\title{
Incidence Of Dry Eye Disesase In People Living With Acquired Immuodeficiency Synrome
}

\author{
Dr.L.J.Sandhyavali, M.S ${ }^{(1)}$; Dr.K.Parimala Devi ${ }^{(2)}$, M.S; Dr.Saketa.K; ${ }^{(3)}$ \\ Dr.K.Sree Divya. ${ }^{(3)}$ \\ ${ }^{I}$ Associate Professor,Department of Ophthalmology, Guntur medical college, Guntur. \\ ${ }^{2}$ Assistant Professor, Department of Ophthalmology, Guntur medical college, Guntur. \\ ${ }^{3}$ Post Graduate, Department of Ophthalmology, Guntur medical college, Guntur.
}

\begin{abstract}
Purpose: To study the incidence of Dry eye syndrome using in people living with Acquired Immunodeficiency syndrome(AIDS), attending ART centre in Government General hospital(GGH), Guntur. Study design: Hospital based prospective study.

Methodology: This study was conducted in 100 patients attending ART centre in GGH, Guntur for a period of 3 months from January 2016 to March 2016. Every patient was subjected to a dry eye questionnaire, anterior segment examination by slit lamp biomicroscopy, tearfilm breakup time(TBUT) and Schirmer's test, were done. Results : Out of 100 people living with AIDS, examined with Schirmer's test 79\% had normal study, mild dry eye in $9 \%$, moderate dry eye in $9 \%$, severe dry eye in $3 \%$ of patients. Out of 100 people examined with tear film breakup test, $82 \%$ had TBUT $<10$ sec and $18 \%$ had TBUT $>10$ sec.

Conclusion: $50 \%$ of people living with AIDS had Dry Eye Disease. Out of which $82 \%$ had lipid and mucin layer deficiency associated with posterior blepharitis. And 18\% had acqeous layer of tear film deficiency.
\end{abstract}

Keywords: Schirmer's test, acqeous tear film deficiency, tear film breakup time.

\section{Introduction}

This study was conducted in 100 patients attending ART centre in GGH, Guntur for a period of 3 months from January 2016 to March 2016. After taking detailed history, anterior segment examination by slit lamp, Schirmer's test was done.

\section{Materials And Methods}

This study was conducted in 100 patients attending ART centre in GGH, Guntur for a period of 3 months from January 2016 to March 2016. Every patient was subjected to a dry eye questionnaire, anterior segment examination by slit lamp biomicroscopy, tearfilm breakup time(TBUT) and Schirmer's test, were done. A widely used method involves instillation of fluorescein dye in the lower conjunctival sac using a fluoresceinimpregnated strip wet with nonpreserved saline solution. The nonpreserved drop is important because preservatives such as benzalkonium chloride (BAC) can artificially speed up tear breakup. After the dye has been distributed throughout the tear film by blinking, the patient is asked to stare straight ahead without blinking. Under slit-lamp examination, the time between the last blink and the appearance of the first break (randomly distributed dry spot or hole) in the precorneal fluorescent tear film is measured ${ }^{(5)}$.

\section{Inclusion criteria:}

Patients diagnosed as HIV positive.

Patients of HIV on HAART (Highly Active Anti Retroviral Therapy).

Patients of age groups from 10yrs to 80 yrs including males and females.

Patients with no other ocular abnormalities.

\section{Exclusion criteria:}

Normal individuals.

Patients with collagen vascular disease, connective tissue disease.

Patients with debilitating illness.

Patients with lagophthalmos.

Patients with burns and cicatricial ectropion.

Patients operated for cataract and refractive surgeries.

Results

According to Schirmer's test,out of 100 patients 79 were normal with out dry eye. 9 patients with mild dry eye. 9 patients with moderate dry eye. 3 patients with severe dry eye. 


\begin{tabular}{|l|l|l|}
\hline Severity & No: of patients & Percent \\
\hline Normal & 79 & $79 \%$ \\
\hline Mild & 9 & $9 \%$ \\
\hline Moderate & 9 & $9 \%$ \\
\hline Severe & 3 & $3 \%$ \\
\hline Total & 100 & 100 \\
\hline
\end{tabular}

Out of 100 patients 52 were females, 48 were males.

\begin{tabular}{|l|l|l|}
\hline Patients & Males & Females \\
\hline 100 & 48 & 52 \\
\hline
\end{tabular}

Out of 52 females 33 patients had normal study, mild dry eye in 6 patients, moderate dry eye in 6 patients, severe dry eye in 3 patients.

\begin{tabular}{|l|l|l|}
\hline Severity & patients & Percentage \\
\hline Normal & 33 & $64 \%$ \\
\hline Mild & 6 & $11.7 \%$ \\
\hline Moderate & 9 & $17.6 \%$ \\
\hline Severe & 3 & $5.8 \%$ \\
\hline Total & 52 & \\
\hline
\end{tabular}

Among 48 male patients severity of dry eye was as following:

\begin{tabular}{|l|l|l|}
\hline Severity & patients & Percentage \\
\hline Normal & 33 & $68.75 \%$ \\
\hline Mild & 3 & $6.25 \%$ \\
\hline Moderate & 9 & $18.75 \%$ \\
\hline Severe & 3 & $6.25 \%$ \\
\hline Total & 48 & \\
\hline
\end{tabular}

Tear film break up time:

Among 100 patients studied, 82 patients had tear film break up time less than $10 \mathrm{sec}, 19$ patients had, TBUT>10 sec.

\begin{tabular}{|l|l|l|}
\hline TBUT & $<10 \mathrm{sec}$ & $>10 \mathrm{sec}$ \\
\hline & 82 & 18 \\
\hline
\end{tabular}

Among 100 patients 52 females, 48 males.

Out of 52 females, 41 patients $(78.8 \%)$ had TBUT $<10$ SEC, out of 48 males, 41 patients $(85.4 \%)$ had TBUT $<10$ SEC.

Schirmers test:

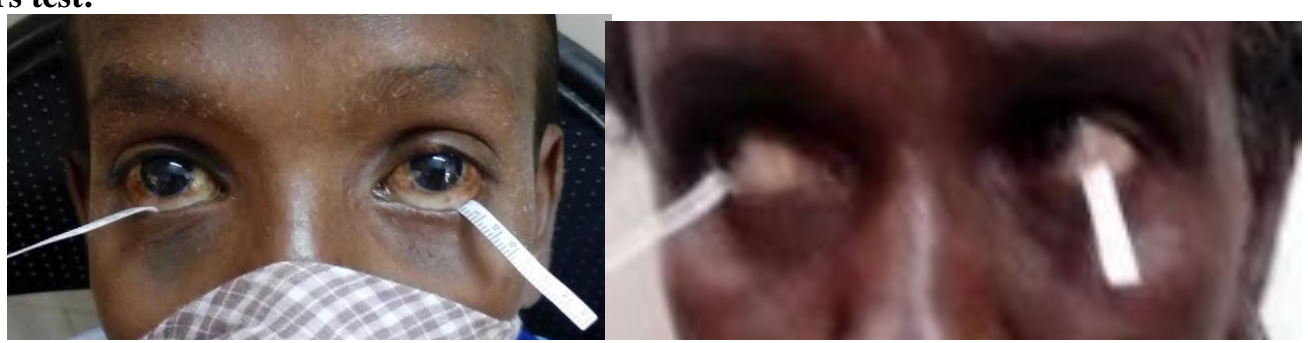

Tear film break up time:

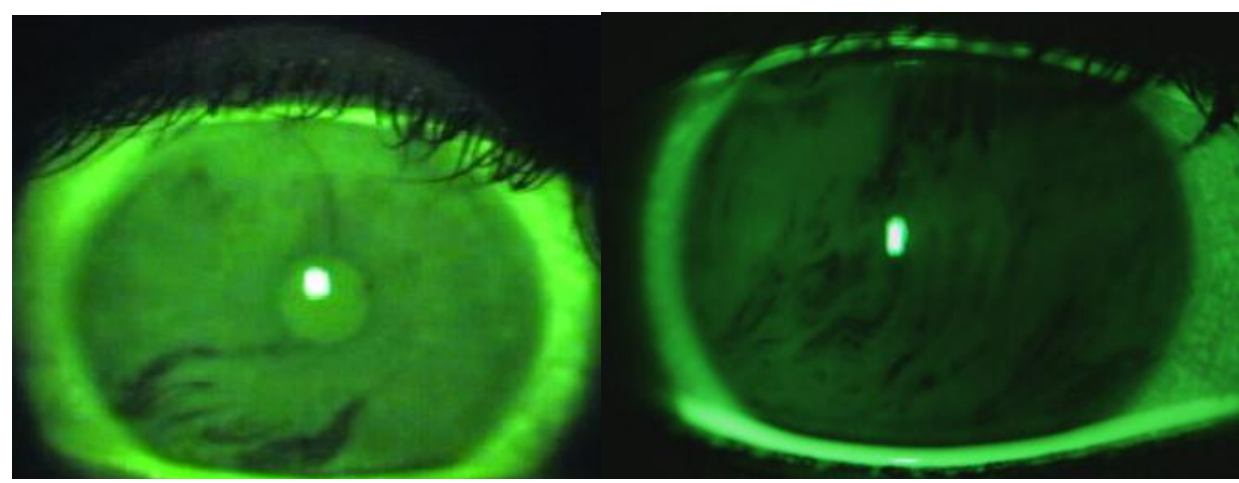




\section{Discussion}

Dry eye disease (DED), or keratoconjunctivitis sicca (KCS), is one of the most common ophthalmologic conditions $^{(1)}$. According to the International Dry Eye WorkShop (DEWS) in 2007,dry eye is defined as a multifactorial disease of the tears and ocular surface that results in symptoms of discomfort, visual disturbance, and tear film instability with potential damage to the ocular surface ${ }^{(2)}$. It is accompanied by increased osmolarity of the tear film and inflammation of the ocular surface. According to epidemiologic studies in the US, the prevalence of clinically diagnosed DED is $0.4-0.5 \%$ overall,and is highest among women and the elderly. Tens of millions more have less severe symptoms and probably a more episodic manifestation of the disease that is notable only during adverse contributing conditions, such as low humidity or contact lens wear. Thus, DED is considered a significant public health problem which will have a considerable economic impact in terms of both direct and indirect costs.

\section{Pathophysiology:}

Maintaining a healthy and comfortable ocular surface requires stability and renewal of the preocular tear film. DED is a multifactorial disorder involving multiple interacting mechanisms. Dysfunction of any component by causing alterations in the volume, composition, distribution, and/or clearance of the tear film can lead to ocular surface disease that expresses itself as dry eye. Two mutually reinforcing global mechanisms, tear hyperosmolarity and tear film instability, have been identified ${ }^{(2)}$. Any subclass of dry eye activates these core mechanisms and explains the features of various forms of dry eye.

Regardless of the initiating event or etiology, inflammation is usually a key factor in perpetuating DED. Chronic inflammation may subsequently result in lacrimal gland insufficiency, reduced corneal sensation (longterm effects of inflammatory mediators on sensory nerve terminals supplying the ocular surface $^{(3)}$, and morphological changes in the sub-basal nerve plexus, and decreased reflex activity including reflex tearing and blinking, leading to increased evaporation and tear film instability. These postulated interactions, occurring over time, may explain the overlap of findings in dry eyes regardless of the underlying etiology, and reinforce the general concept of a vicious circle in which widely varying influences combine to cause dry eye with a complex profile ${ }^{(2)}$.

\section{Role of hiv in ded:}

HIV (human immunodeficiency virus) infection may present with a large variety of primary and secondary (caused by opportunistic infections) ocular manifestations, some of those have threatening potential to vision and life quality. The incidence and presentation of the AIDS epidemic and its correlated complications have dramatically changed since the introduction of the potent antiretroviral therapies (also know as highly active antiretroviral therapy, or HAART), but remains as a dramatic public health in very low income countries.

DED appears to be much more prevalent among individuals with AIDS (21.4-38.8\% of HIV-infected men, $16.9 \%$ of HIV-infected women) than in the general population ${ }^{(11)}$. Burtin et al. evaluated the ocular surface and DED complains in a group of HIV positive patients. According to this study, $70 \%$ of them had complained of DED symptoms, $85 \%$ present at least one clinical sign of ocular surface dysfunction tested through Schirmer test, tear break-up time and lissamine stain and the impression cytology revealed a decrease in the number of dendritic cells ${ }^{(12)}$.

Geier et al. showed that decreased tear production occurs in approximately $20 \%$ to $25 \%$ of patients with HIV infection without correlation with the CD4+ lymphocites blood count. Although the entire pathogenesis of the aqueous tear deficiency in HIV-infected remain unclear, it may be associated with lymphocytic infiltration and destruction of the lacrimal gland acini and ducts.

In fact, a Sjögren's syndrome-like picture may be present in HIV -infected patients who develop the diffuse infiltrative lymphocytosis syndrome (DILS). Thus, it is an exclusion criteria for individuals under investigation for Sjögren syndrome ${ }^{(13)}$. DILS is a disorder in patients with HIV infection that is characterized by the enlargement of salivary and lacrimal glands and a varying intensity of DED symptoms. In addition, DILS is accompanied by persistent circulating and infiltration of CD8-positive lymphocytes. DILS may mimic Sjögren's syndrome in terms of symptoms and parotid and lacrimal glands involvement. On the other hand, it differs by the high frequency of extra glandular sites of lymphocytic infiltration, such as lungs, muscles and liver, scarcity or absence of serum autoantibodies and the nature of infiltrating lymphocytes. While in Sjögren syndrome presents lymphocytic infiltration of CD4+ and in DILS it is a CD8+ lymphocytes and anti-Ro and anti-La are seen less frequently ${ }^{(14)}$. The prevalence of DILS had dropped significantly after the introduction of HAART, indirectly inferring that the HIV infection contributes to DILS pathogenesis. 


\section{Etiopathogenic classification:}

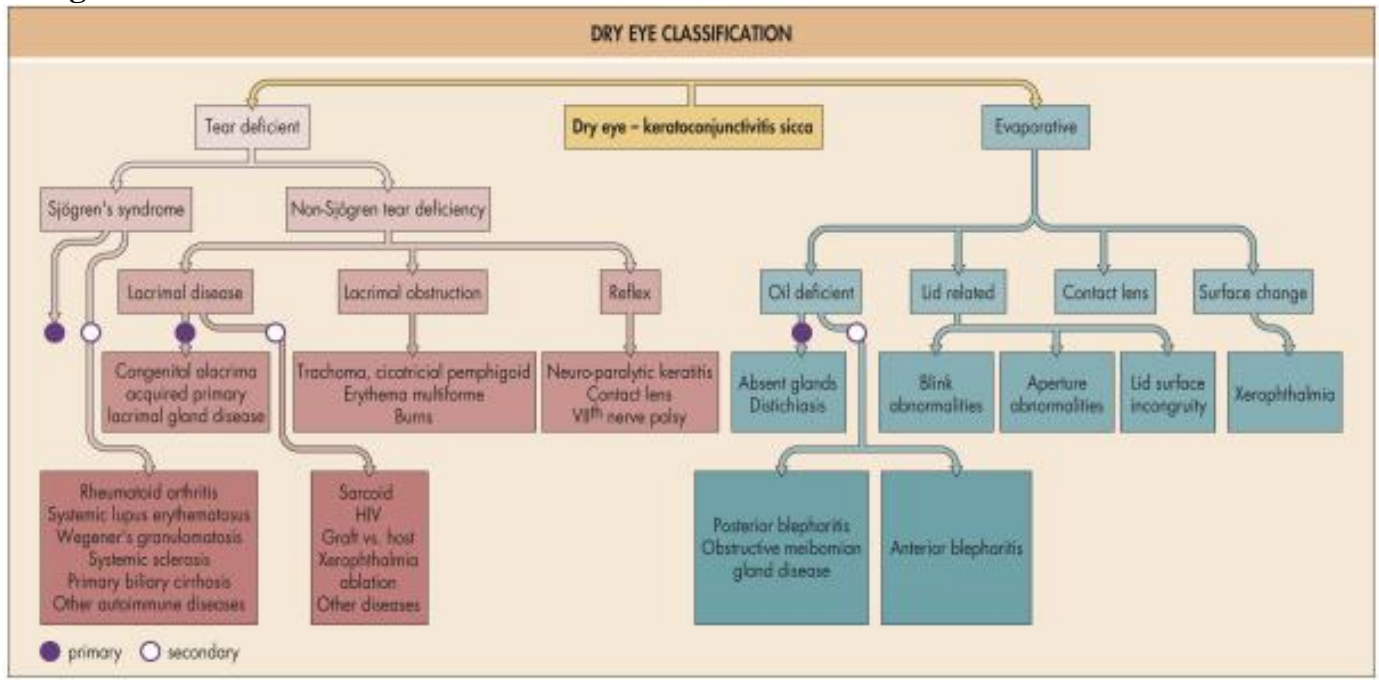

Diagnostic workup:

Traditionally, combinations of questionnaires and diagnostic tests have been used to assess symptoms and clinical signs. The combination of history and examination must be used because some patients have significant symptoms but few findings, whereas others have significant clinical findings with only mild symptoms.

Subjective symptoms of dry eye are the hallmark of this disease. There may be any number of symptoms, such as foreign body sensation, burning, stinging, itching, dryness, soreness, heaviness of the lids, photophobia, and ocular fatigue. The symptoms reported by patients with dry eyes share some common patterns. An important clue is exacerbation of symptoms by certain activities or environmental condition. Fluctuating vision during such activities is an extremely common complaint. Intolerance of the drift from air conditioners, smoky environments, and the low humidity of airplane cabins are other helpful clues when making the diagnosis of dry eye. In general, patients with aqueous tear deficiency tend to get worse as the day progresses, whereas those with predominantly meibomian gland disease are worse in the morning. Similarly, a symptomatic response to artificial tears supports the diagnosis of dry eye. A number of questionnaires are available for evaluating various aspects of dry disease symptomatology, including severity, effect on daily activities, and quality of life.

\section{Examination:}

It begins by evaluating the face and eyelids for signs of rosacea or floppy lids. The dynamics of blinking and lid position should be observed while taking the history to prevent conscious alterations.

Points of interests are: a) frequency of blinking; b) variation of blink intervals; c) size of the palpebral aperature, and d) adequacy of lid closure. Malposition of the lids may influence tear spread and turnover; therefore look for: a) entropion; b) ectropion; c) eversion of the lacrimal puncta; d) cicatrical malposition; e) dermatochalasis; and f) swelling of the temporal aspect of the upper lid, which may imply enlargement of the lacrimal gland.

Slit lamp examination: a) Lid margins: hyperemia, telangiectasia, thickening, scarring, keratinization, ulceration, tear debris, abnormalities of the meibomian orifices, including metaplasia, and the character of expressed meibomian secretions. b) Eyelashes: misdirection, malposition, encrustations, collarettes, and staphylococcal blepharitis; c) Conjunctiva: erythema, swelling, keratinization, papillary/follicular reaction, pinguecula, lid parallel conjunctival folds (conjunctival chalasis); d) Cornea: infiltrates, scars, punctate staining or ulcers, vascularization, pannus, and pterygium. In addition, the tear film should be analyzed for filaments, mucus, and cellular debris and foam. Corneal sensation should likewise be examined in all patients.

In many patients with mild to moderate dry eye the slit lamp examination may not be revealing and further diagnostic testing is needed to evaluate and stage patients more objectively.

\section{Diagnostic tests:}

Sequence of tests to be done in dry eye includes the following:

1. Flouroscein strip with small non preserved saline drop:

a) Measure tear break up time

b) Look for the staining of cornea 
2. Lissamine strip with small non preserved saline drop

a) Look for staining of conjunctiva

3. Dry excess tears in the eye cul-de-sac

4. Place Schirmers strips in both eye with no anesthesia: measure wetness after 5 minutes.

5. Measure corneal sensations.

\section{Tear stability testing:}

Tear film stability, which is reduced in all forms of dry eye, is commonly evaluated by performing a tear breakup time (TBUT) test. A widely used method involves instillation of fluorescein dye in the lower conjunctival sac using a fluorescein-impregnated strip wet with nonpreserved saline solution. The nonpreserved drop is important because preservatives such as benzalkonium chloride (BAC) can artificially speed up tear breakup. Place a small drop of nonpreserved saline over the proximal end of the fluorescein strip and let it flow towards the tip. The drop is gently applied to the inferior tarsal conjunctiva. The patient should be instructed to blink several times to disperse the dye. After the dye has been distributed throughout the tear film by blinking, the patient is asked to stare straight ahead without blinking. Under slit-lamp examination, the time between the last blink and the appearance of the first break (randomly distributed dry spot or hole) in the precorneal fluorescent tear film is measured ${ }^{(5)}$.

Alternatively, tear film stability can be measured in a noninvasive fashion using Tearscope or keratometry devices.

Fluorescein TBUT has been reported to be rapid in different types of dry eye, including keratoconjunctivitis sicca, mucin deficiency, and meibomian gland disease. There is wide variability in the tear breakup time of normal subjects, but an arbitrary cutoff time of $10 \mathrm{~s}$ for both fluorescein-added and noninvasive techniques appears quite specific in screening patients for evidence of tear film instability however, cutoffs as low as $<5$ s have also been recommended.

Fluorescein sodium dye is currently the most commonly used. When the surface epithelial cells loosen or desquamate, the dye diffuses rapidly in the intercellular spaces and staining indicates increased epithelial permeability. It also penetrates the corneal epithelium when the mucous layer is removed. Fluorescein generally stains the cornea to a greater degree than the conjunctiva.

\section{Schirmers test:}

The most commonly used technique for measuring tear secretion is the Schirmer test. The Schirmer I test is classically done without anesthesia and measures reflex tearing. Van Bijsterveld ${ }^{(6)}$ selected a cutoff value of $5.5 \mathrm{~mm}$ strip wetting in 5 minutes for the Schirmer test without anesthesia to diagnose aqueous tear deficiency. Using this cutoff, he reported that the correct diagnosis was made in $83 \%$ of dry eye patients tested. Furthermore, those with measures of $6-10 \mathrm{~mm}$ and $>10 \mathrm{~mm}$ were considered as dry eye suspect and normal, respectively. If the Schirmer test is performed after nasal stimulation, it is termed the Schirmer II test. In contrast to non-Sjögren dry eye, in Sjögren's syndrome it has been shown that the ability of nasal stimulation to increase the tear production of the anesthetized eye is greatly reduced, a finding of diagnostic value ${ }^{(7)}$

Jones $^{(8)}$ popularized the use of topical anesthesia with a Schirmer test as a measurement of basal tear secretion, independent of reflex tearing. Generally, $3-5 \mathrm{~mm}$ of wetting is used as the cutoff when performing 'Schirmer with anesthesia'. In general, for patients with more severe dry eyes such as those with Sjögren's syndrome, it is best to perform the classic Schirmer I test (without anesthesia) to obtain a measure of the patient's reserve of lacrimal function.

The test is performed by placing a Schirmer strip in the lateral one-third of the lower lid. It is important to dry the fornix before placing the strip and to ask the patient to refrain from talking or chewing gum during the procedure. If using an anesthetic, adequate time should be given after the drop to minimize reflex tearing from the burning sensation due to the drop.

It is most useful in diagnosing patients with more severe dry eyes who have significant aqueous tear deficiency, but not very useful as a screening test, particularly in patient with mild aqueous deficiency. A more generous cutoff of $10 \mathrm{~mm}$ can be used to indicate aqueous tear deficiency. However, the patients with less than $10 \mathrm{~mm}$ can be further segregated to document the extent of aqueous tear deficiency. This is especially helpful when trying to differentiate Sjögren's and non-Sjögren's etiologies. Specifically, in patients with Sjögren's syndrome, the Schirmer is usually $<5 \mathrm{~mm}$.

\section{Conclusion}

The tear film is the most dynamic structure of the functional unit, and its production and turnover is essential to maintaining the health of the ocular surface. A dysfunction of any of these layers can result in dry eye disease. DED appears to be much more prevalent among individuals with AIDS than in the general population. 


\section{References}

[1]. Krachmer > Volume 1 - Fundamentals and Medical Aspects of Cornea and External Disease > Part V - The Ocular Adnexa > Section 4 - Disorders of Tear Production and the Lacrimal System > Chapter 36 - Dry Eye.

[2]. The definition and classification of dry eye disease: report of the Definition and Classification Subcommittee of the International Dry Eye WorkShop (2007). Ocul Surf 2007; 5:75-92

[3]. Bourcier T, Acosta MC, Borderie V, et al: Decreased corneal sensitivity in patients with dry eye. Invest Ophthalmol Vis Sci 2005; 46:2341-2345

[4]. Ophthalmology- Myron Yanoff and Jay S Duker $-4^{\text {th }}$ edition - Part 4 - Section 6 - page no: 325

[5]. Lemp MA: Report of the National Eye Institute/Industry Workshop on clinical trials in dry eyes. CLAO J 1995; 21:221-232

[6]. van Bijsterveld OP: Diagnostic tests in the Sicca syndrome. Arch Ophthalmol 1969; 82:10-14

[7]. \Methodologies to diagnose and monitor dry eye disease: report of the Diagnostic Methodology Subcommittee of the International Dry Eye WorkShop (2007). Ocul Surf 2007; 5:108-152

[8]. Jones LT: The lacrimal secretory system and its treatment. Am J Ophthalmol 1966; 62:47-60.

[9]. Kanski's clinical ophthalmology a systemic approach, $8^{\text {th }}$ edition, chapter 4, pages 119-130.

[10]. Parson's diseases of the eye - 22th edition - pages 188,189

[11]. DeCarlo DK, Penner SL, Schamerloh RJ, Fullard RJ. Dry eye among males infected with the human immunodeficiency virus. J Am Optom Assoc. 1995;66(9):533-8.

[12]. Burtin T, Guepratte N, Bourges JL, Garcher C, Le Hoang P, Baudouin C. [Abnormalities of the ocular surface in patients with AIDS]. J Fr Ophtalmol. 1998;21(9):637-42. French

[13]. Vitali C. Classification criteria for Sjögren's syndrome. Ann Rheum Dis. 2003;62(1):94-5; author reply 95

[14]. Kordossis T, Paikos S, Aroni K, Kitsanta P, Dimitrakopoulos A, Kavouklis E, et al. Prevalence of Sjögren's-like syndrome in a cohort of HIV-1-positive patients: descriptive pathology and immunopathology. Br J Rheumatol. 1998;37(6):691-5. 